\title{
H3K27me3 induces multidrug resistance in small cell lung cancer by affecting HOXA1 DNA methylation via regulation of the IncRNA HOTAIR
}

\author{
Shun Fang ${ }^{1 \#}$, Yefeng Shen ${ }^{2 \#}$, Bin Chen ${ }^{3}$, Yuanzhou $\mathrm{Wu}^{2}$, Longfei Jia ${ }^{2}$, Yaling $\mathrm{Li}^{2}$, Yaru Zhu ${ }^{2}$, Yusheng Yan ${ }^{2}$, \\ Man $\mathrm{Li}^{1}$, Rui Chen ${ }^{1}$, Linlang Guo ${ }^{1}$, Xin Chen ${ }^{4}$, Qunqing Chen ${ }^{2}$ \\ ${ }^{1}$ Department of Pathology, ${ }^{2}$ Department of Cardiothoracic Surgery, Zhujiang Hospital, Southern Medical University, Guangzhou 510282, China; \\ ${ }^{3}$ Department of Hepatic Surgery, The First Affiliated Hospital, Sun Yat-sen University, Guangzhou 510080, China; ${ }^{4}$ Department of Pulmonary and \\ Critical Care Medicine, Zhujiang Hospital, Southern Medical University, Guangzhou 510282, China \\ Contributions: (I) Conception and design: Q Chen, S Fang; (II) Administrative support: Q Chen, S Fang; (III) Provision of study materials or patients: \\ Y Shen, B Chen, Y Wu, L Jia; (IV) Collection and assembly of data: Y Shen, B Chen, Y Li, Y Zhu, Y Yan; (V) Data analysis and interpretation: M Li, \\ R Chen, L Guo, X Chen; (VI) Manuscript writing: All authors; (VII) Final approval of manuscript: All authors. \\ \#These authors contributed equally to this work. \\ Correspondence to: Shun Fang, MD, PhD. Department of Pathology, Zhujiang Hospital, Southern Medical University, 253 Gongye Road, Guangzhou \\ 510282, China. Email: function1945@163.com; Qunqing Chen, MD, PhD. Department of Cardiothoracic Surgery, Zhujiang Hospital, Southern \\ Medical University, 253 Gongye Road, Guangzhou 510282, China. Email: chenqqg1985@sina.com.
}

Background: The long non-coding RNA (lncRNA) HOX transcript antisense RNA (HOTAIR) serves as a powerful predictor of tumor progression and overall survival in patients. Our previous studies showed that HOTAIR modulated HOXA1 DNA methylation by reducing DNMT1 and DNMT3b expression in drugresistant small cell lung cancer (SCLC). Moreover, H3 lysine 27 trimethylation (H3K27me3) is catalyzed by enhancer of zeste homolog 2 (EZH2) and plays a critical role in SCLC chemoresistance. However, it is not completely clear whether $\mathrm{H} 3 \mathrm{~K} 27 \mathrm{me} 3$ affects HOXA1 DNA methylation or whether this effect is mediated by HOTAIR.

Methods: The levels of EZH2 and H3K27me3 were identified in SCLC tissues by immunohistochemical (IHC) staining and in SCLC multidrug-resistant cells by Western blotting. Cell counting kit-8 (CCK-8) and flow cytometry were used to detect and analyze the biological function of $\mathrm{H} 3 \mathrm{~K} 27 \mathrm{me} 3$. Then, we assessed the role of HOTAIR in the regulation of $\mathrm{EZH} 2$ and $\mathrm{H} 3 \mathrm{~K} 27 \mathrm{me} 3$ by using lentivirus and small interfering RNA. Further, bisulfite sequencing PCR was conducted to detect the methylation levels of HOXA1 DNA. Finally, Western blotting was performed to examine the regulatory role of $\mathrm{H} 3 \mathrm{~K} 27 \mathrm{me} 3$ in controlling HOTAIR expression in SCLC.

Results: In this study, we found that EZH2 and H3K27me3 levels were markedly higher in SCLC tissues and multidrug-resistant SCLC cells. The results indicated that $\mathrm{H} 3 \mathrm{~K} 27 \mathrm{me} 3$ was related to multidrug resistance. HOTAIR overexpression and knockdown showed that EZH2 and $\mathrm{H} 3 \mathrm{~K} 27 \mathrm{me} 3$ were regulated by HOTAIR. Moreover, H3K27me3 affected HOXA1 DNA methylation levels. Strikingly, we found that H3K27me3 acted as a negative feedback regulator of HOTAIR.

Conclusions: Our study showed that H3K27me3 affects HOXA1 DNA methylation via HOTAIR regulation, indicating that $\mathrm{H} 3 \mathrm{~K} 27 \mathrm{me} 3$ may be a potential therapy target for SCLC chemoresistance.

Keywords: H3K27me3; HOTAIR; DNA methylation; chemoresistance; small cell lung cancer (SCLC)

Submitted May 01, 2018. Accepted for publication Sep 25, 2018.

doi: $10.21037 /$ atm.2018.10.21

View this article at: http://dx.doi.org/10.21037/atm.2018.10.21 


\section{Introduction}

Lung cancer, with approximately 1.8 million new cases and more than 1 million related deaths each year, is the most common malignancy and has rapidly emerged as a primary cause of cancer-related death worldwide over the past several decades (1). Small cell lung cancer (SCLC) is the most aggressive type of lung cancer and accounts for approximately $15-18 \%$ of all lung cancer cases (2). The majority of SCLC patients are initially responsive to chemotherapy drugs, but they inevitably develop multidrug resistance within a year $(3,4)$. Thus, a better understanding of the mechanisms underlying multidrug resistance is urgently needed for improving SCLC treatment.

HOX transcript antisense RNA (HOTAIR), a widely studied long non-coding RNA (lncRNA), is transcribed from the HOXC gene cluster, bridges polycomb repressive complex 2 (PRC2) and lysine-specific demethylase 1 (LSD1) and suppresses the transcription of HOXD genes by catalyzing $\mathrm{H} 3$ lysine 27 trimethylation (H3K27me3) and the spontaneous demethylation of H3K4 (5-7). Many researchers have also demonstrated that HOTAIR and enhancer of zeste homolog 2 (EZH2) interact with each other in different types of cancer, inducing gene silencing and influencing chromatin dynamics (7-11). Moreover, aberrant DNA methylation has been demonstrated to participate in the development of chemoresistance in various types of cancer, such as ovarian and lung cancer (12). In our previous study, HOTAIR knockdown decreased HOXA1 DNA methylation by reducing the expression of DNMT1 and DNMT3b in chemoresistant SCLC (13).

Histone methylation is a vital epigenetic phenomenon that participates in a diverse array of cellular processes, such as transcription control, RNA splicing and tumorigenesis (14-19). $\mathrm{H} 3 \mathrm{~K} 27 \mathrm{me}$, which is catalyzed by EZH2 (20), is correlated with transcriptional repression, is essential for cancer chemoresistance and is a prognostic factor in lung cancer $(21,22)$. Multiple studies have shown that HOTAIR couples with EZH2 to mediate both cell invasion and cell metastasis $(23,24)$. However, whether HOTAIR affects HOXA1 DNA methylation in SCLC by regulating EZH2mediated $\mathrm{H} 3 \mathrm{~K} 27 \mathrm{me} 3$ remains largely unknown.

In this study, we found that EZH2 and $\mathrm{H} 3 \mathrm{~K} 27 \mathrm{me} 3$ enrichment levels were markedly high in SCLC tissues and multidrug-resistant SCLC cells. Moreover, H3K27me3 was related to multidrug resistance. We found that HOTAIR promoted EZH2 and EZH2-mediated H3 K2 7 me 3. $\mathrm{H} 3 \mathrm{~K} 27 \mathrm{me} 3$ affected the methylation level of HOXA1.
Furthermore, H3K27me3 acted as a feedback regulator of HOTAIR. Thus, these results suggest that H3K27me3 induces multidrug resistance in SCLC by affecting HOXA1 DNA methylation via the regulation of the lncRNA HOTAIR.

\section{Methods}

\section{Cell lines and cell culture}

The human SCLC cell lines NCI-H69 and NCI-H446 and the multidrug-resistant subline NCI-H69AR were purchased from the American Type Culture Collection (ATCC). Cells were cultured in RPMI 1640 containing $10 \%$ fetal bovine serum (Gibco, CA, USA) at $37{ }^{\circ} \mathrm{C}$ in a humidified incubator with $5 \% \mathrm{CO}_{2}$.

\section{Immunobistochemical (IHC) staining}

Formalin-fixed, paraffin-embedded tissues were sectioned, deparaffinized and rehydrated gradually. Sample sections were incubated with primary antibodies directed against EZH2 (Cell Signaling Technology, \#5426, USA) and H3K27me3 (Cell Signaling Technology, \#9733, USA) at $4{ }^{\circ} \mathrm{C}$ overnight. Secondary antibodies were applied according to the manufacturer's protocol. The percentage of positive cells was calculated in five randomly selected high-power fields. The results were classified based on the total scores of the percentage of positively stained tumor cells and the staining intensity. The percentages of positively stained tumor cells were classified into four groups with scores ranging from 0 to $3(<10 \%, 0 ;>10 \%, 1 ;>25 \%, 2$; and $>50 \%, 3)$. The staining intensities were scored as follows: no staining, 0 ; low, 1; medium, 2; and high, 3). A final IHC score was calculated by adding the two scores; a score $>3$ indicated positive expression, and a score $\leq 3$ indicated negative expression.

\section{Western blotting}

Protein was extracted from cells with RIPA lysis buffer, and the protein concentrations were detected using the standard BCA method (BCA ${ }^{\mathrm{TM}}$ Protein Assay Kit, CoWin Biosciences, China). Subsequently, each protein sample was separated on $12 \%$ SDS-PAGE gels and transferred onto PVDF membranes (Millipore, Etten-Leur, Netherlands). After blocking with $5 \%$ nonfat milk for $2 \mathrm{~h}$, the membranes were incubated with the primary antibodies used for IHC at 
$4{ }^{\circ} \mathrm{C}$ overnight. The membranes were then incubated with a horseradish peroxidase-labeled secondary antibody. Finally, the protein bands were visualized using chemiluminescence (ECL) after being washed again with TBST.

\section{Quantitative real-time polymerase chain reaction (qRT-PCR)}

Total RNA was extracted from the cell lines with TRIzol reagent (Invitrogen, NY, USA) according to the manufacturer's instructions. RT-PCR assays were performed to detect HOTAIR expression using a Prime Script RT reagent kit (TIANGEN, Beijing, China) according to the manufacturer's protocol. The primers used in our study were as follows: HOTAIR, (F) 5'-GGTAGAAAAAGCAACCACGAAGC-3' and (R) 5'-ACATAAACCTCTGTCTGTGAGTGCC-3'; GAPDH, (F) 5'-GAGTCAACGGATTTGGTCGT-3' and (R) 5'-CATGGGTGGAATCATATTGGA-3'. Data collection was performed with an ABI Illumina instrument. The results were normalized to the expression of GAPDH. The relative expression of $\mathrm{A}$ was analyzed using the $2^{-\Delta \Delta \mathrm{Ct}}$ method.

\section{Bisulfite sequencing PCR (BSP)}

Cell DNA was isolated, bisulfate modified, and detected as previously described (20). The primer sequences for PCR were as follows: (F) 5'-GT TTAGTTTTAGAATAGAGGAGGTGGTT-3' and (R) 5'-AAAATCAATAAACCAA AACTC-3'. The region of the D promoter is shown in Figure $S 1$ in the supplementary material.

\section{Flow cytometry}

Cells were treated with chemotherapy drugs for $24 \mathrm{~h}$ and collected for cell apoptosis and cell cycle analyses. Cell apoptosis assays were performed using an Annexin V eFluor $^{\text {TM }} 450 /$ eFluor $^{\text {TM }} 660$ kit (eBioscience, San Diego, USA) according to the manufacturer's protocol. For cell cycle analysis, cells were fixed in $70 \%$ ethanol at $4{ }^{\circ} \mathrm{C}$ overnight. Subsequently, the cells were incubated with RNase and stained with Fixable Viability Dye eFluor ${ }^{\mathrm{TM}}$ 660. Finally, CellQuest Pro software was used for apoptosis analyses, and ModFit LT software was used for cell cycle analyses.

\section{Cell counting kit-8 (CCK-8)}

Cells were seeded in 96 -well plates at $7 \times 10^{3}$ cells per well. Following culture for $6 \mathrm{~h}$, the cells were treated with chemotherapy drugs [cisplatin (CDDP; Shandong, China), etoposide (VP-16; Jiangsu, China) and adriamycin (ADM; Jiangsu, China)] for $24 \mathrm{~h}$. The absorbance at $450 \mathrm{~nm}$ was measured after incubation with $10 \mu \mathrm{L}$ of CCK- 8 reagent (Dojindo, Kumamoto, Japan) for approximately 4 h. Cells without chemotherapy drug treatment were used to indicate $100 \%$ survival. The assay was conducted in six replicate wells for each sample, and three parallel experiments were performed.

\section{Cell transfection}

For stable transfection, H69 and H446 cells were infected with HOTAIR lentiviral particles (GenePharma, Shanghai, China) and control lentiviruses according to the manufacturer's instructions. For HOTAIR knockdown, cells were transfected separately with two siRNAs (GenePharma, Shanghai, China) using Lipofectamine 2000 (Invitrogen, NY, USA). The transfection efficiencies were detected by qRT-PCR. The siRNAs used were as follows: siHOTAIR\# 1, 5'-CCCAUGGACUCAUAAACAATT-3' and 5 '- UUGUUUAUGAGUCCAUGGGTT-3 '; siHOTAIR\#2, 5'-GC CUUUGGAAGCUCUUGAATT-3' and 5'-UUCAAGAGCUUCCAAAGGCTT-3'.

\section{Statistical analysis}

All experiments were performed in triplicate. The data are shown as the means $\pm \mathrm{SD}$, and the statistical analyses were carried out with SPSS 22.0 software. Student's $t$-test was used to evaluate the difference between two means, and comparisons between more than two groups were calculated using one-way ANOVA. $\mathrm{P}<0.05$ was considered statistically significant $\left({ }^{*} \mathrm{P}<0.05,{ }^{*} \mathrm{P}<0.01\right)$.

\section{Results}

\section{H3K27me3 induced multidrug resistance in SCLC}

To determine if $\mathrm{H} 3 \mathrm{~K} 27 \mathrm{me} 3$ is associated with SCLC, we performed $\mathrm{IHC}$ to detect $\mathrm{H} 3 \mathrm{~K} 27 \mathrm{me} 3$ expression in five SCLC tissue samples. As EZH2 was previously demonstrated to catalyze H3K27 methylation (25), we also examined the expression of EZH2. H3K27me3 and EZH2 
were detected predominantly in the cell nucleus. EZH2 and $\mathrm{H} 3 \mathrm{~K} 27 \mathrm{me} 3$ levels were markedly higher in SCLC cells than in normal lung alveolar epithelial cells (Figure 1A).

Then, we assessed H3 K27me3 in H69AR/H69 and H446 cells with Western blotting assays and found that the $\mathrm{H} 3 \mathrm{~K} 27 \mathrm{me} 3$ levels were increased in the multidrug-resistant cells compared with those in the parental cells (Figure $1 B$ and Figure S2). We used highly selective inhibitors to alter the $\mathrm{H} 3 \mathrm{~K} 27 \mathrm{me} 3$ levels. EI1, a selective inhibitor of EZH2, inhibits EZH2/PRC2 methyltransferase activity and decreases $\mathrm{H} 3 \mathrm{~K} 27$ methylation without changing other histone $\mathrm{H} 3$ methylation marks $(25,26)$. GSK J4 is a selective inhibitor of two demethylases, JMJD3/UTX, the inhibition of which increases H3K27 methylation levels $(27,28)$. EI1treated cells (H69AR cells) exhibited decreased H3K27me3 upon EZH2 downregulation; in addition, GSK J4-treated cells (H69 and H446 cells) exhibited increased H3K27me3 with unchanged EZH2 levels (Figure $1 C$ and Figure S2). CCK-8 assays indicated that the IC50 values of CDDP, ADM and VP-16 were significantly decreased in H69AR cells after treatment with EI1 and that the IC50 values significantly increased in H69 and H446 cells with GSK J4 treatment (Figure 1D). Flow cytometry showed that the apoptosis rates significantly increased in EI1-treated cells after anticancer drug treatment, whereas the apoptosis rates significantly decreased in GSK J4-treated cells (Figure 1E). Furthermore, cell cycle progression was not significantly changed (Figure S3). Thus, all these data indicate that $\mathrm{H} 3 \mathrm{~K} 27 \mathrm{me} 3$ induces multidrug resistance and exerts antiapoptotic effects in SCLC.

\section{EZH2 and H3K27me3 were upregulated by HOTAIR}

To determine whether HOTAIR plays a key role in EZH2 and $\mathrm{H} 3 \mathrm{~K} 27$ trimethylation, we examined the enrichment levels of EZH2 and $\mathrm{H} 3 \mathrm{~K} 27 \mathrm{me} 3$ under HOTAIR interference. We previously found that HOTAIR mRNA levels were higher in H69AR cells than in H69 and H446 cells (13). We then used a lentivirus to overexpress HOTAIR by nearly 658 -fold in H69 cells and 4340-fold in H446 cells (Figure $2 A$ ). HOTAIR overexpression significantly increased H3K27me3 and EZH2 levels in H69 and H446 cells according to Western blotting assays (Figure 2B). In contrast, HOTAIR expression was downregulated by as much as $70 \%$ in H69AR cells transfected with two different siRNAs (Figure 2C). Strikingly, silencing HOTAIR significantly reduced the levels of both EZH2 and H3K27me3 (Figure 2D). Collectively, these data suggest that $\mathrm{EZH} 2$ and $\mathrm{H} 3 \mathrm{~K} 27 \mathrm{me} 3$ are upregulated by HOTAIR.

\section{HOTAIR affected HOXA1 DNA metbylation through H3K27me3}

To explore whether HOTAIR affects HOXA1 DNA methylation by regulating $\mathrm{H} 3 \mathrm{~K} 27 \mathrm{me} 3$, we used BSP to investigate the methylation status of the $\mathrm{CpG}$ island in the HOXA1 promoter. The analysis revealed that HOTAIR overexpression resulted in increased $\mathrm{H} 3 \mathrm{~K} 27 \mathrm{me} 3$ levels and CpG island methylation levels in the HOXA1 promoter in H69 and H446 cells. In contrast, HOTAIR knockdown resulted in decreased $\mathrm{H} 3 \mathrm{~K} 27 \mathrm{me} 3$ levels and $\mathrm{CpG}$ island methylation levels in the HOXA1 promoter in H69AR cells (Figure 3A). Next, we found that H3K27me3 levels decreased with EI1 treatment and that HOXA1 DNA methylation levels decreased consistently with $\mathrm{H} 3 \mathrm{~K} 27$ me3 downregulation in HOTAIR-overexpressing cells (H69 and H446). Conversely, H3K27me3 levels increased with GSK J4 treatment and HOXA1 DNA methylation levels increased consistently with $\mathrm{H} 3 \mathrm{~K} 27 \mathrm{me} 3$ upregulation in HOTAIR-knockdown cells (H69AR) (Figure 3B). These results indicate that $\mathrm{H} 3 \mathrm{~K} 27 \mathrm{me} 3$ affects HOXA1 DNA methylation via HOTAIR regulation.

\section{H3K27me3 acted as a negative feedback regulator of HOTAIR}

To further identify the association between $\mathrm{H} 3 \mathrm{~K} 27 \mathrm{me} 3$ and HOTAIR, we investigated whether HOTAIR expression is dependent on $\mathrm{H} 3 \mathrm{~K} 27 \mathrm{me} 3$ levels. $\mathrm{H} 3 \mathrm{~K} 27 \mathrm{me} 3$ was dramatically increased in H69 and H446 cells treated with GSK J4 according to Western blotting assays (Figure $1 C$ and Figure $4 A$ ). This increase occurred in a dosedependent manner. However, after treatment with the same concentration of GSK J4, qRT-PCR indicated that HOTAIR expression levels constantly increased and reached a peak of $3.0 \mu \mathrm{M}$ in $\mathrm{H} 69$ cells and of $1.5 \mu \mathrm{M}$ in H446 cells and then decreased (Figure 4B). These results indicate that $\mathrm{H} 3 \mathrm{~K} 27 \mathrm{me} 3$ acts as a negative feedback regulator of HOTAIR.

\section{Discussion}

SCLC has been designated a "recalcitrant" cancer due to its high lethality and the lack of substantial therapeutic progress made over the past years. Recent studies have revealed that the IncRNA HOTAIR acts as a vital mediator of the 
A
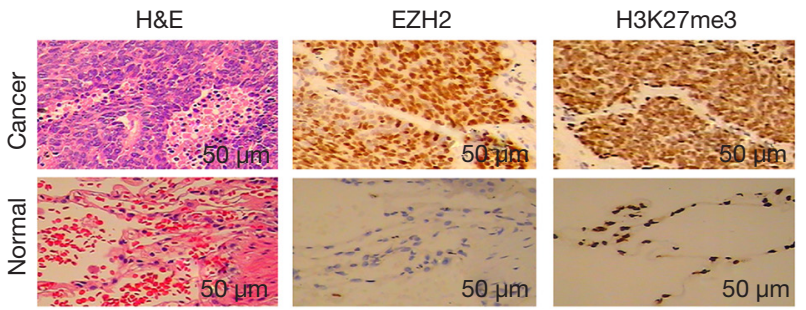

C

H69AR

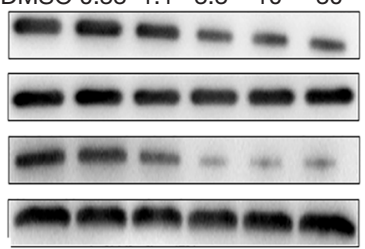

D

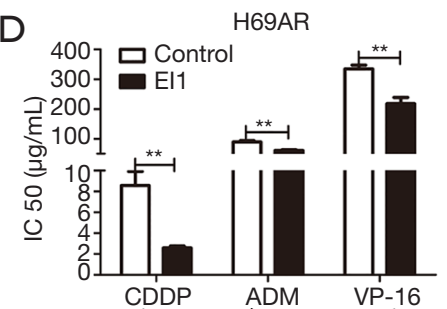

$\mathrm{E}$

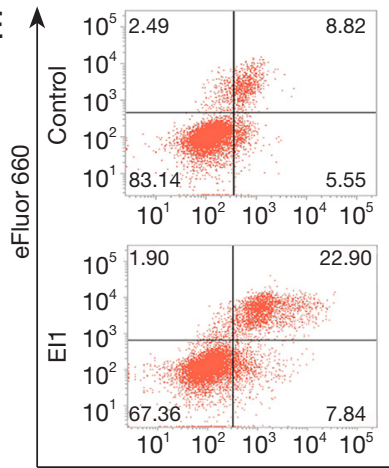

H69AR

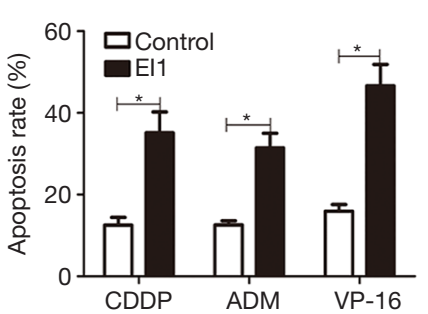

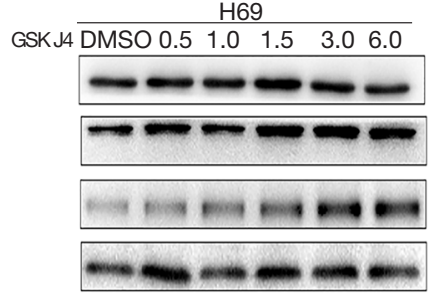

H69
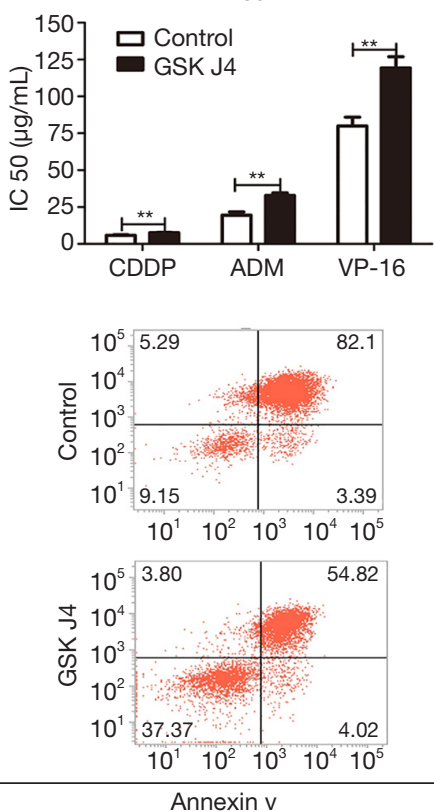

H69

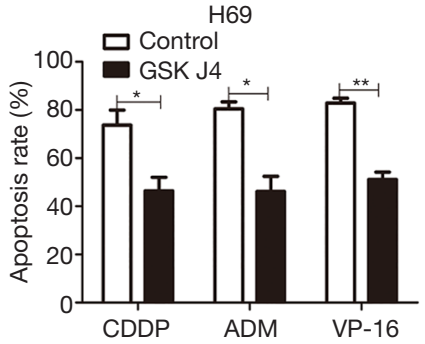

B
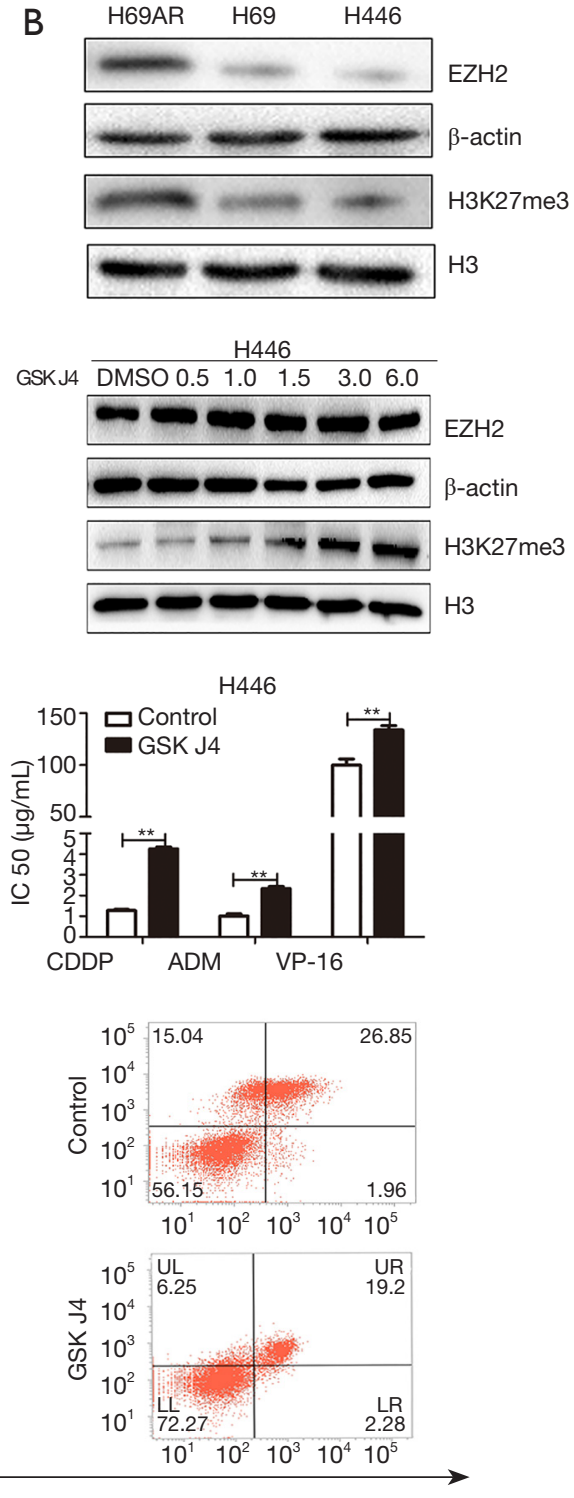

H446

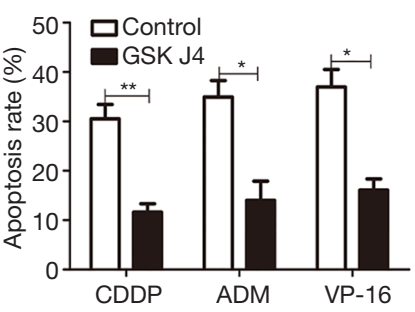

Figure $1 \mathrm{H} 3 \mathrm{~K} 27 \mathrm{me} 3$ was related to resistance to anticancer drug treatment. (A) H3K27me3 and EZH2 levels in SCLC tissue and normal lung tissue were detected by IHC staining; (B) H3K27me3 and EZH2 levels in multidrug-resistant cells and sensitive cells were detected by Western blotting; (C) H3K27me3 and EZH2 levels after treatment with different concentrations of EI1 and GSK J4; (D) IC50 values were measured with CCK-8 assays after EI1-treated and GSK J4-treated cells were exposed to CDDP, ADM, and VP-16; (E) cell apoptosis was determined by flow cytometric analysis in EI1-treated and GSK J4-treated cells after drug exposure. The results are presented as the mean \pm SD. ${ }^{*} \mathrm{P}<0.05,{ }^{* *} \mathrm{P}<0.01$, compared with the control group. 
A
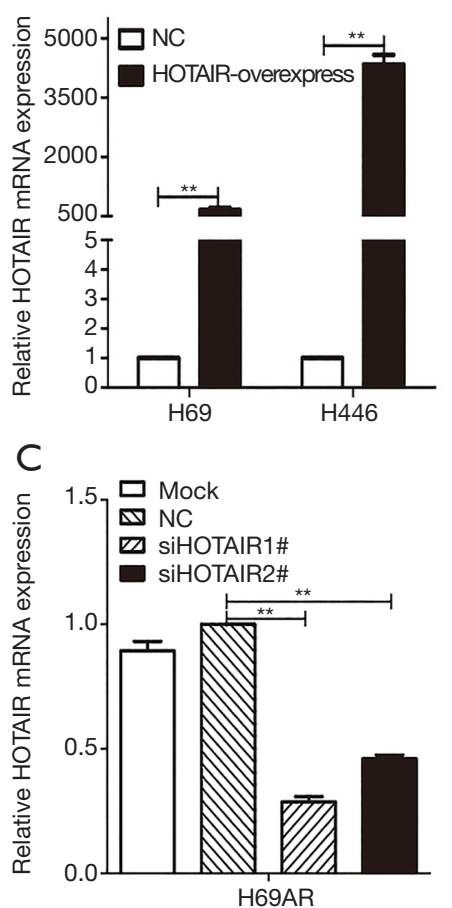

B
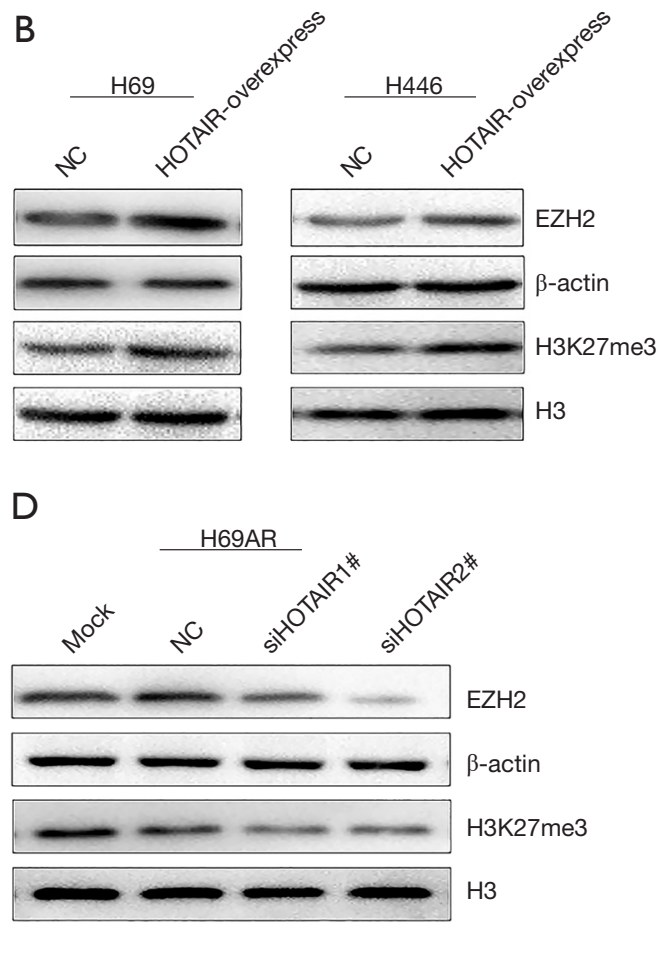

Figure 2 EZH2 and H3K27me3 levels were upregulated by HOTAIR. (A,C) HOTAIR overexpression and knockdown were validated by RT-PCR; (B,D) H3K27me3 and EZH2 enrichment were measured with Western blotting assays of the HOTAIR-overexpressing and HOTAIR-knockdown cells. The results are presented as the mean $\pm \mathrm{SD}$. ${ }^{* *} \mathrm{P}<0.01$, compared with the control group.

molecular mechanisms underlying cancer development and progression, including proliferation, metastasis and chemoresistance $(13,23,29,30)$. Our previous studies showed that HOTAIR regulates HOXA1 DNA methylation by reducing DNMT1 and DNMT3b levels in chemoresistant SCLC. Moreover, HOTAIR couples with EZH2 to act as a pivotal mediator of cell invasion and metastasis. EZH2 possesses histone methyltransferase activity and methylates H3K27 (26,31). Recently, mounting evidence has suggested that $\mathrm{H} 3 \mathrm{~K} 27 \mathrm{me} 3$ is correlated with chemoresistant cancer, including SCLC $(21,22)$. To verify the hypothesis that $\mathrm{H} 3 \mathrm{~K} 27 \mathrm{me} 3$ affects HOXA1 DNA methylation via HOTAIR regulation in SCLC chemoresistance, we initially found that EZH2 and H3K27me3 levels were markedly higher in SCLC tissues than in adjacent noncancerous tissues. Furthermore, we found that EZH2 and H3K27me3 were upregulated in multidrug-resistant SCLC cells. To further validate the biological functions of $\mathrm{H} 3 \mathrm{~K} 27 \mathrm{me} 3$, we determined the effect of $\mathrm{H} 3 \mathrm{~K} 27 \mathrm{me} 3$ on SCLC cell sensitivity to anticancer drugs. Our data indicated that H3K27me3 affects multidrug resistance by inhibiting apoptosis in SCLC. However, previous studies have shown that $\mathrm{H} 3 \mathrm{~K} 27 \mathrm{me} 3$ suppresses proliferation via apoptosis and cell cycle arrest in cancer $(32,33)$. This inconsistency may be attributed to the different types of cancer.

To further investigate whether EZH2 and H3K27me3 are regulated by HOTAIR, we successfully established two stable HOTAIR-overexpressing cell sublines and effectively suppressed HOTAIR expression using siRNAs. HOTAIR overexpression significantly increased EZH2 and H3K27me3 levels, whereas HOTAIR knockdown significantly reduced both EZH2 and H3K27me3 levels. Our results indicate that EZH2 and $\mathrm{H} 3 \mathrm{~K} 27 \mathrm{me} 3$ are upregulated by HOTAIR.

CpG island methylation in the promoter may be a crucial mechanism regulating gene expression at the epigenetic level $(34,35)$. Moreover, histone modifications alter DNA methylation $(36,37)$. HOTAIR has been found to bind PRC2, mediate $\mathrm{H} 3 \mathrm{~K} 27$ trimethylation and silence the HOXD locus (38). To investigate whether HOTAIR affects HOXA1 DNA methylation through H3K27me3, we determined the correlation between the CpG island 
A

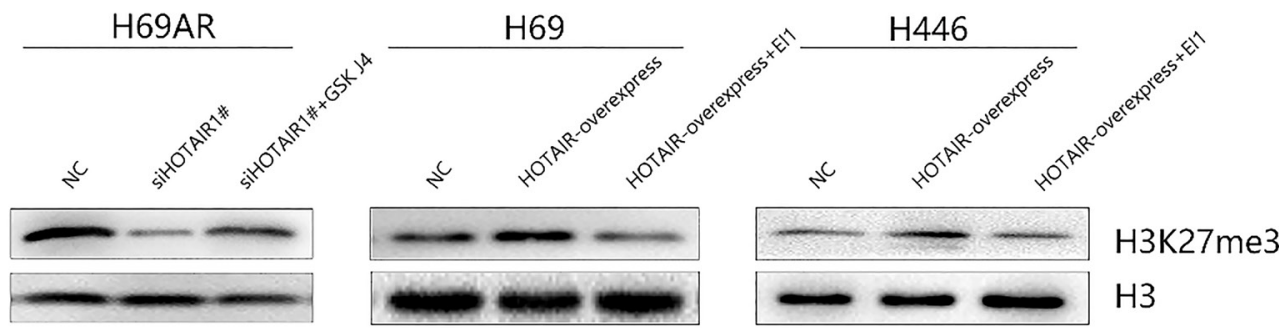

B

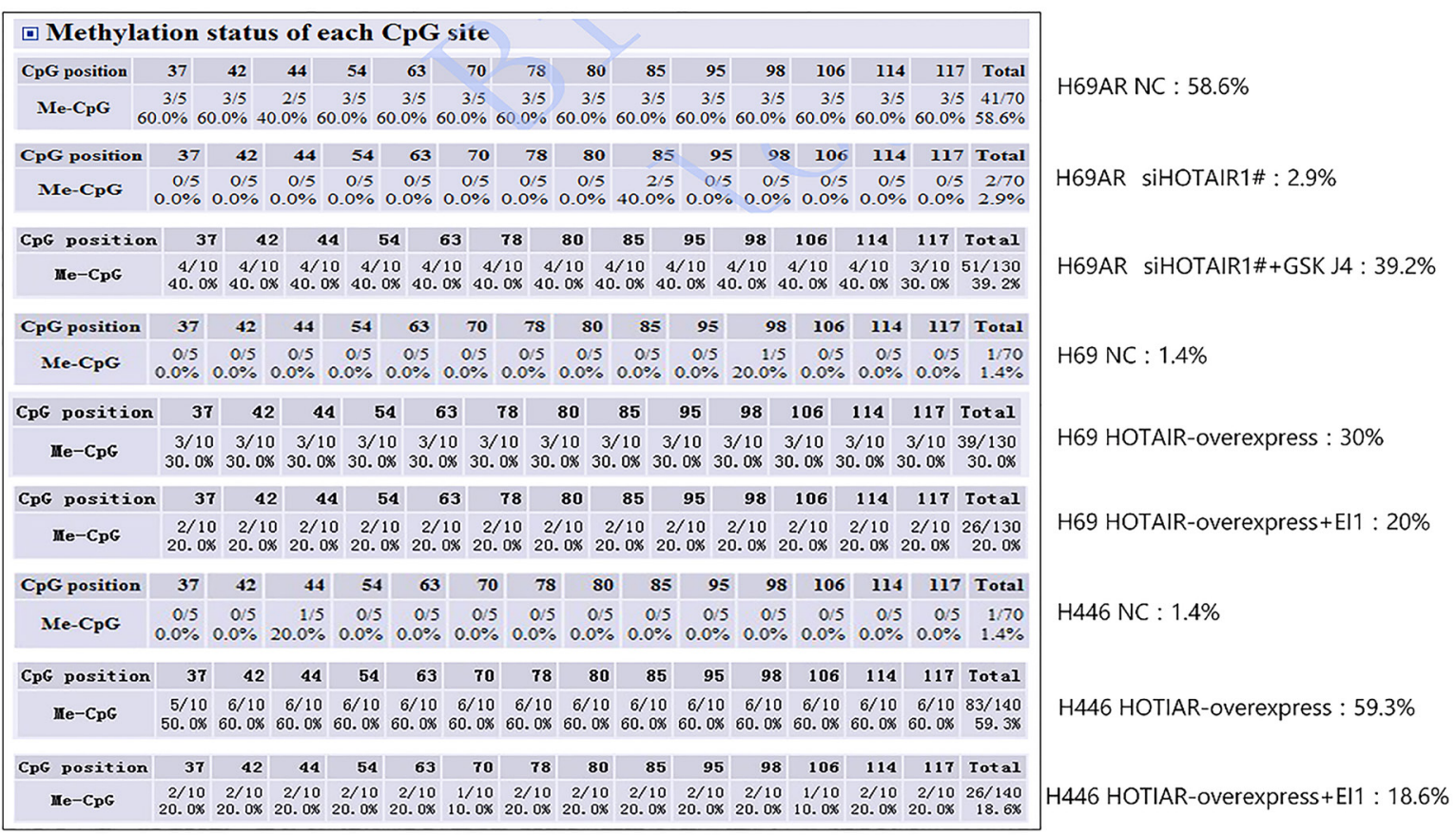

Figure 3 H3K27me3 affected HOXA1 DNA methylation via HOTAIR regulation. (A) The levels of H3K27me3 in HOTAIRknockdown cells treated with GSK J4 and HOTAIR-overexpressing cells treated with EI1 determined by Western blotting assays; (B) analysis of the HOXA1 CpG islands in HOTAIR-knockdown cells treated with GSK J4 and HOTAIR-overexpressing cells treated with EI1 according to BSP.

methylation status and H3K27me3 levels. HOXA1 DNA methylation levels decreased consistently with $\mathrm{H} 3 \mathrm{~K} 27 \mathrm{me} 3$ downregulation and increased consistently with $\mathrm{H} 3 \mathrm{~K} 27 \mathrm{me} 3$ upregulation, which validated our hypothesis. However, the molecular mechanisms involved in $\mathrm{H} 3 \mathrm{~K} 27 \mathrm{me} 3$-induced HOXA1 DNA methylation need to be further explored in the future.

A previous study indicated that EZH2-mediated H3K27 methylation contributes to the repression of a subset of lncRNAs in embryonic stem cells (10). Our study provides evidence to support the hypothesis that $\mathrm{H} 3 \mathrm{~K} 27 \mathrm{me} 3$ may repress HOTAIR expression. HOTAIR expression constantly increased and reached its peak level and then decreased dependent on increasing levels of $\mathrm{H} 3 \mathrm{~K} 27 \mathrm{me} 3$. Additionally, H3K27me3 can bind the HOTAIR promoter region in human liver cancer stem cells (39). Therefore, all the results indicate that $\mathrm{H} 3 \mathrm{~K} 27 \mathrm{me} 3$ suppresses HOTAIR, likely by binding to its promoter. To the best of our knowledge, this is the first study to reveal a negative feedback loop between H3K27me3 and HOTAIR in SCLC cells. This information may aid in understanding the pleiotropic functions of $\mathrm{H} 3 \mathrm{~K} 27$ methylation in the 
A

$\mathrm{H} 69$

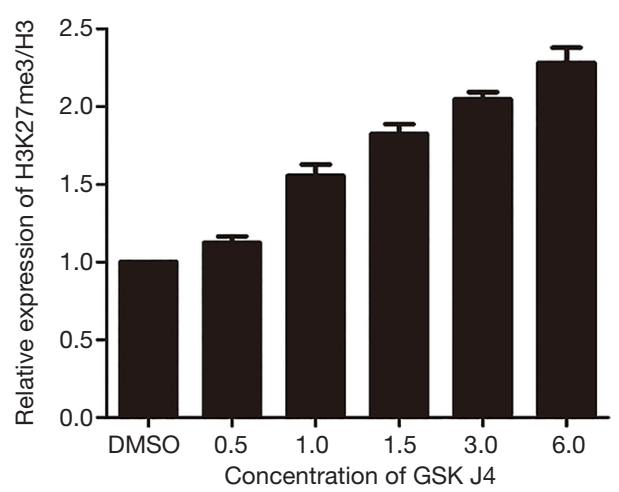

B

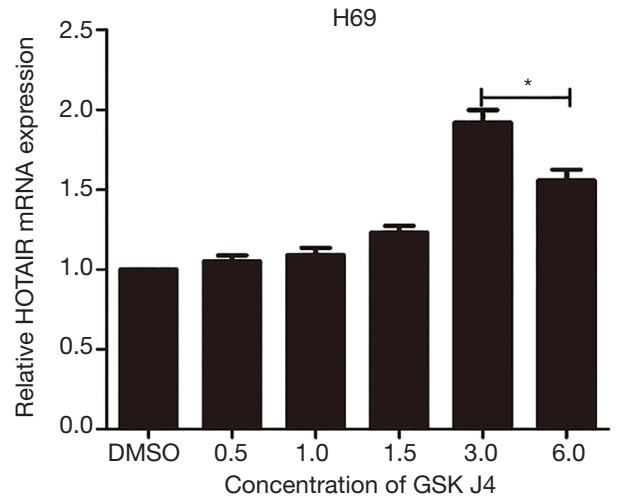

$\mathrm{H} 446$
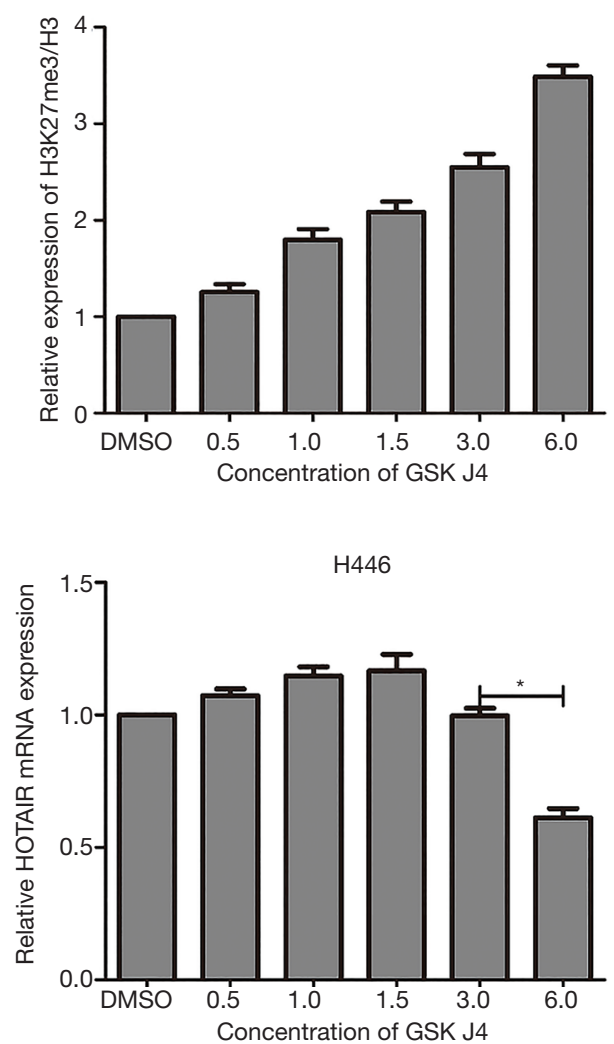

Figure 4 H3K27me3 may repress HOTAIR expression. (A) Relative expression of H3K27me3/H3 upon treatment with different concentrations of GSK J4 (0, 0.5, 1, 1.5, 3.01, and 6.01 $\mathrm{M})$; (B) HOTAIR mRNA expression upon treatment with different concentrations of GSK J4 $(0,0.5,1,1.5,3.01$, and $6.01 \mu \mathrm{M})$. The results are presented as the mean $\pm \mathrm{SD}$. ${ }^{*} \mathrm{P}<0.05$, compared with the control group.

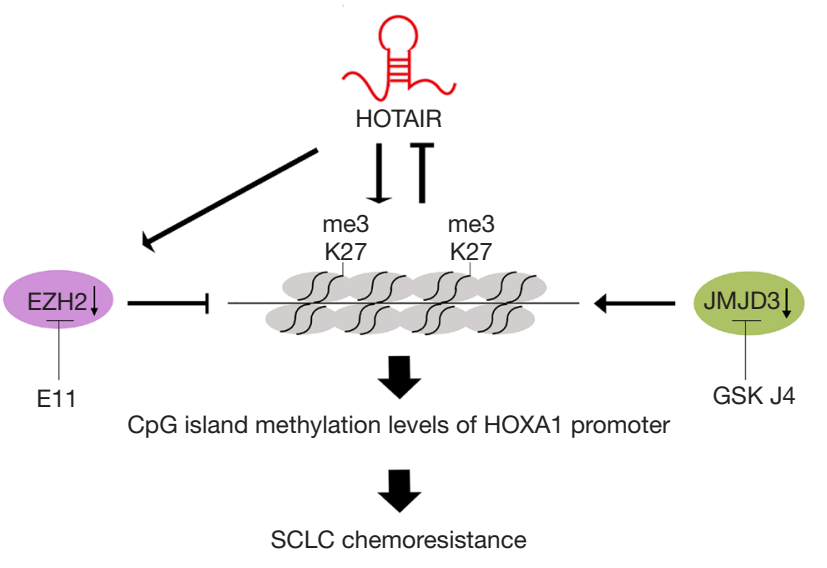

Figure 5 Schematic illustration of HOTAIR/EZH2-mediated H3K27me3 and HOXA1 DNA methylation in chemoresistant SCLC cells. regulation of lncRNA expression in cancer progression. However, further experiments need to be performed to determine the mechanism by which H3K27me3 suppresses HOTAIR expression.

\section{Conclusions}

In conclusion, our study found that $\mathrm{H} 3 \mathrm{~K} 27 \mathrm{me} 3$ induced multidrug resistance in SCLC by affecting HOXA1 DNA methylation via regulation of the lncRNA HOTAIR. Moreover, H3K27me3 modulated HOTAIR expression through a negative feedback mechanism (Figure 5). Therefore, our study aided in understanding the mechanism of SCLC chemoresistance and indicated that H3K27me3 may be a potential therapeutic target for treating chemoresistance in SCLC, but this treatment must be 
confirmed with additional clinical studies.

\section{Acknowledgements}

Funding: This work was supported by the Natural Science Foundation of Guangdong Province (2016A030310385) and the National Natural Science Foundation of China (81601986).

\section{Footnote}

Conflicts of Interest: The authors have no conflicts of interest to declare.

\section{References}

1. Torre LA, Bray F, Siegel RL, et al. Global cancer statistics, 2012. CA Cancer J Clin 2015;65:87-108.

2. Planchard D, Le Pechoux C. Small cell lung cancer: new clinical recommendations and current status of biomarker assessment. Eur J Cancer 2011;47 Suppl 3:S272-83.

3. Semenova EA, Nagel R, Berns A. Origins, genetic landscape, and emerging therapies of small cell lung cancer. Genes Dev 2015;29:1447-62.

4. Kalemkerian GP, Schneider BJ. Advances in Small Cell Lung Cancer. Hematol Oncol Clin North Am 2017;31:143-56.

5. Spizzo R, Almeida MI, Colombatti A, et al. Long noncoding RNAs and cancer: a new frontier of translational research? Oncogene 2012;31:4577-87.

6. Wahlestedt C. Targeting long non-coding RNA to therapeutically upregulate gene expression. Nat Rev Drug Discov 2013;12:433-46.

7. Gupta RA, Shah N, Wang KC, et al. Long non-coding RNA HOTAIR reprograms chromatin state to promote cancer metastasis. Nature 2010;464:1071-6.

8. Tsai MC, Manor O, Wan Y, et al. Long non-coding RNA as modular scaffold of histone modification complexes. Science 2010;329:689-93.

9. Kogo R, Shimamura T, Mimori K, et al. Long non-coding RNA HOTAIR regulates polycomb-dependent chromatin modification and is associated with poor prognosis in colorectal cancers. Cancer Res 2011;71:6320-6.

10. Wu SC, Kallin EM, Zhang Y. Role of H3K27 methylation in the regulation of lncRNA expression. Cell Res 2010;20:1109-16.

11. Wu L, Murat P, Matak-Vinkovic D, et al. Binding interactions between long non-coding RNA HOTAIR and
PRC2 proteins. Biochemistry 2013;52:9519-27.

12. Teschendorff AE, Lee SH, Jones A, et al. HOTAIR and its surrogate DNA methylation signature indicate carboplatin resistance in ovarian cancer. Genome Med 2015;7:108.

13. Fang S, Gao H, Tong $\mathrm{Y}$, et al. Long non-coding RNAHOTAIR affects chemoresistance by regulating HOXA1 methylation in small cell lung cancer cells. Lab Invest 2016;96:60-8.

14. Strahl BD, Allis CD. The language of covalent histone modifications. Nature 2000;403:41-5.

15. Greer EL, Shi Y. Histone methylation: a dynamic mark in health, disease and inheritance. Nat Rev Genet 2012;13:343-57.

16. Kahn TG, Schwartz YB, Dellino GI, et al. Polycomb complexes and the propagation of the methylation mark at the Drosophila ubx gene. J Biol Chem 2006;281:29064-75.

17. Vermeulen M, Eberl HC, Matarese F, et al. Quantitative interaction proteomics and genome-wide profiling of epigenetic histone marks and their readers. Cell 2010;142:967-80.

18. Chi P, Allis CD, Wang GG. Covalent histone modifications--miswritten, misinterpreted and mis-erased in human cancers. Nat Rev Cancer 2010;10:457-69.

19. Albert M, Helin K. Histone methyltransferases in cancer. Semin Cell Dev Biol 2010;21:209-20.

20. Ibanez de Caceres I, Cortes-Sempere M, Moratilla C, et al. IGFBP-3 hypermethylation-derived deficiency mediates cisplatin resistance in non-small-cell lung cancer. Oncogene 2010;29:1681-90.

21. Gardner EE, Lok BH, Schneeberger VE, et al. Chemosensitive Relapse in Small Cell Lung Cancer Proceeds through an EZH2-SLFN11 Axis. Cancer Cell 2017;31:286-99.

22. Avila-Moreno F, Armas-Lopez L, Alvarez-Moran AM, et al. Overexpression of MEOX2 and TWIST1 is associated with $\mathrm{H} 3 \mathrm{~K} 27 \mathrm{me} 3$ levels and determines lung cancer chemoresistance and prognosis. PLoS One 2014;9:e114104.

23. Wu Y, Zhang L, Zhang L, et al. Long non-coding RNA HOTAIR promotes tumor cell invasion and metastasis by recruiting EZH2 and repressing E-cadherin in oral squamous cell carcinoma. Int J Oncol 2015;46:2586-94.

24. Zheng J, Xiao X, Wu C, et al. The role of long non-coding RNA HOTAIR in the progression and development of laryngeal squamous cell carcinoma interacting with EZH2. Acta Otolaryngol 2017;137:90-8.

25. Qi W, Chan H, Teng L, et al. Selective inhibition of Ezh2 by a small molecule inhibitor blocks tumor 
cells proliferation. Proc Natl Acad Sci U S A 2012;109:21360-5.

26. Cao R, Wang L, Wang H, et al. Role of histone $\mathrm{H} 3$ lysine 27 methylation in Polycomb-group silencing. Science 2002;298:1039-43.

27. Xiang Y, Zhu Z, Han G, et al. JMJD3 is a histone H3K27 demethylase. Cell Res 2007;17:850-7.

28. Yan N, Xu L, Wu X, et al. GSKJ4, an H3K27me3 demethylase inhibitor, effectively suppresses the breast cancer stem cells. Exp Cell Res 2017;359:405-14.

29. Sun L, Fang J. Writer meets eraser in HOTAIR. Acta Biochim Biophys Sin (Shanghai) 2011;43:1-3.

30. Woo CJ, Kingston RE. HOTAIR lifts non-coding RNAs to new levels. Cell 2007;129:1257-9.

31. Morey L, Helin K. Polycomb group protein-mediated repression of transcription. Trends Biochem Sci 2010;35:323-32.

32. Pawlyn C, Bright MD, Buros AF, et al. Overexpression of EZH2 in multiple myeloma is associated with poor prognosis and dysregulation of cell cycle control. Blood Cancer J 2017;7:e549.

33. Zhang K, Sun X, Zhou X, et al. Long non-coding RNA HOTAIR promotes glioblastoma cell cycle progression in an EZH2 dependent manner. Oncotarget 2015;6:537-46.

Cite this article as: Fang S, Shen $\mathrm{Y}$, Chen B, Wu Y, Jia L, Li Y, Zhu Y, Yan Y, Li M, Chen R, Guo L, Chen X, Chen Q. $\mathrm{H} 3 \mathrm{~K} 27 \mathrm{me} 3$ induces multidrug resistance in small cell lung cancer by affecting HOXA1 DNA methylation via regulation of the IncRNA HOTAIR. Ann Transl Med 2018;6(22):440. doi: 10.21037/atm.2018.10.21
34. Kumegawa K, Maruyama R, Yamamoto E, et al. A genomic screen for long non-coding RNA genes epigenetically silenced by aberrant DNA methylation in colorectal cancer. Sci Rep 2016;6:26699.

35. Merry CR, Forrest ME, Sabers JN, et al. DNMT1associated long non-coding RNAs regulate global gene expression and DNA methylation in colon cancer. Hum Mol Genet 2015;24:6240-53.

36. Funata $S$, Matsusaka K, Yamanaka R, et al. Histone modification alteration coordinated with acquisition of promoter DNA methylation during Epstein-Barr virus infection. Oncotarget 2017;8:55265-79.

37. Inoue A, Jiang L, Lu F, et al. Maternal H3K27me3 controls DNA methylation-independent imprinting. Nature 2017;547:419-24.

38. Rinn JL, Kertesz M, Wang JK, et al. Functional demarcation of active and silent chromatin domains in human HOX loci by non-coding RNAs. Cell 2007;129:1311-23.

39. An J, Wu M, Xin X, et al. Inflammatory related gene IKKalpha, IKKbeta, IKKgamma cooperates to determine liver cancer stem cells progression by altering telomere via heterochromatin protein 1-HOTAIR axis. Oncotarget 2016;7:50131-49. 
CATTTCTGGGCTCCACATTGCAGAGAAAGTGTTACCTGCGGGTTTTAAATATTTTTCTCCAAAATCAGGCATTTGGA GGCTAATATTTTGCTTTATAAATAGGTTCACCTTATAGAGTTTCCTCTATCCTAATTTCACAGGAAACATCACCAGC AGGCTAATATTTCCTAATGTGGCTAGTGGATAAACATCATACCTAGAGAAGTGTCACACACACATACACACACAA AAATGTACAGAGGCCCCTCAGTTGCATTCCTGCCCACACTCAACAAACACTGCCAATGGTTACACACCACAGAGA CACAAGCTCTAGATGTGTATGTGGTCAGGTTTACTCCATGCAACAGACAAAAGCATATAAATAATATTCCTTCTTA TGTGCACCCATAGGCACACCGAGTTAAATAAAACGTTTGTGTGTGGGCACATGAGGGAATTGTGGGCTGCATATA CACCCTAGTTTGCAAATCTTGTCTCACGACCCGACCCACGTTTACAAATTAGAAATTTAGATACCTAAGCTGCAGG GATTCCTACCATCAAAAATGACAGAACCTAGATTTGATAGTCATGATGCTTTTTCCTTTCATCCAACAGAATTGTAT TGACAAAAATATTTTCTTTGAATTATACAAAGACAGAAAGAGGAGGGAAAGATGAGATGAGTTGTCCTGAACCTC TGCCCCCAAATAACGAATTTGAAACAGAGACCAACGAAGTGCCCTCACTGAAAGCACTGCAAGGGCCATGATTAT CTTTATACAGAAGAAACATGCAGCAAATATAGATGGGTATGGTGACTGTCCCCGCAGGTCATCTCCATCCCCAGTG CCAACCCCTACAAAACAAACCTCTGGGGCTTTCTACTGTGGGACACCCAATGTATAAACATCAGGGTGGCCCCAGT ATAGGCGTCTTGTTGTGTCTGGAAGAGCCTGGCCATTTGTGCCAGAGGGAAAGCAGCCAGGCCCCACCTTCCCACC CCCAGGAGCTGTGGAGAGGAGAGGGAGGGGGCTGGTTTCTCCTTCTGTCCCCTCCTTCCTTAAAAACAGTGTATAA AAATTCCAATACAGATTTCTCCTAGAGAAAAAAAAAAAAAAGCCCTAACCTCCACACTACACATTTAACTGCGAC AGACGGAGACATCTGGCAGAAGGGCGGTGGAGCCAAAAAAATTCCTATAAATTTAAATTCGATGATGGTTACAAT TAACTCCACAACAAACGTGTACTTAAACAGAAAGCCGTTGCTGGGCGTTTGGACACAGCTCGAGCTGCACGTGAG AAAGGAGAGCTTCAGAGTAGCTATGGAATGTCCTCAGCTTGAGAATGTGAGGAGACTACGGCCATACTCTTGGCC CTGCCAGCGGAGAGGAGCTCCAGGCCGGCTAGCACCGGCCTATCTCACCTCTGCCTGCCTGTCCTCACCCGCTGGG CTCTGCCTGCCTGCCTCCTCCTCCTCCGCCGCTGCCATGGGAGCATGGAGGATTTTAAGAGGAGAGGGGTTAATAA AAGGGTCAAATAAAATCATAATATTGAACAGAATGAAATTTATCTTTATTTGCCACCAAAAAAATTTCACAGCAAT CCCTCTGGGAAGGGAGCAGGGTGGGGAGTGCACATGTACACACGCACTCACGCAGAGGAGTAGAGATGCGTGAT TTGAATCTCACTGGGGGCGTCCCCCCCTTTCCCTCCAGGTGGAAAGAAACGCTTGGGAAATTTATTTACAAAAATC CAGGCCCCAGCACGGGGGAGGTGCCCAATCCCATCTCCCTACTGCCTCCACCACCCCAATCTTCAATTTTCTTAAA AAAAAAAAAAAAAGAGGTAACGACTGCATAGACTATAGCTATAAATCCATGAAGTGGGGCACTGCTTGGAAAAA GATGTGGAAACCTCAATGCACCGATTAAGAAATTTCAAAGGAAGGTTCTACACATTCAGGGGGAGAACAACTAGG CTCGGGCGGGGCCTTGGCGAAGACGGAGGTAGGACACGGACTTTGCACACCTAACACCAGGTTGAAGGTACAGA AATTTCACAGCAAGGCAGGATCACATTTAGCTGCAAGGTGGGGCGGGGTAGAGGGGGGAGCTCGAGAAGTTTCCG CTGGCAGACATGCTGTCTTAGCGGATCTTTTCACATGGGCAAGGGAAGGCTTTTAGTTCGAGGATTGTTCGGCTCA GGCGCTCACTACTGTCGCCCCTCCCCCACCTAATCCGAGCAGCAAGACATTGTCGCCGAGGTGTGTTTTCTTTTAA ATCAAAGTCTTGGCCTCGGCGCCAACGGTGTTCACTCGCCGTGCATCAGACACTTCCCTTCACCCCCTTTCCAGGCC CGGTCCCTGTCCCACAGCGAGAGTCAGATAGAGGGTCCAGGAGGTTCCGGAATGGAGTGCCGGCCACAGCGGTGG GGGCCAGCCCTGGCCACAGTCCAGTTTTCCACCGGCGGAAAGAAGACGAGTTAGAAAATAAAAAATAAAAATAA AATAAAATAATTTTCCCCTCCCCCCGCCCCCAAAAGGGCCCGGTCTGCAGGTTACAGCAGAGGATTTCCCGAGAA ATACTGCAGCCGGTCTCTGCTCAGTTTCTTTTCTTTCATCCTTCTGTTCTGAAACCAAATTTTCACTTGTCGGTCCGT CAGGTTCAGCATCCGGGACAGCTGCAGCCGCTTCTCTTTGTTGATATACACGTTGAAGAAAAACTCTCGCTCCAGT TCCCGGATCTGGAATTTCGAATAAGGGCAGCGCTTCTTGCGGGTGCGGGGGGCGTCTGGAGGGAGGGGAGGGGGC AAGTGCGAGGAGAGGGGGGAGAGAGACACGTGAGACCCGGGCGACCCCAGCCCGCTGAGCGCTCAGCGGCCCTT CTCCTAGCCCACCGCGGGCAGCCCTCCCGCCAGGCGGCTGGCTCTCCTGCAGCCGCAGCACCCGGGCAAGCCTCG ACCCCCTGCCAGGTCTAGCGGGTCCCCACCCCCCCGCCACTGCGCCACCCCGTCC

Figure S1 The HOXA1 promoter region. 
A

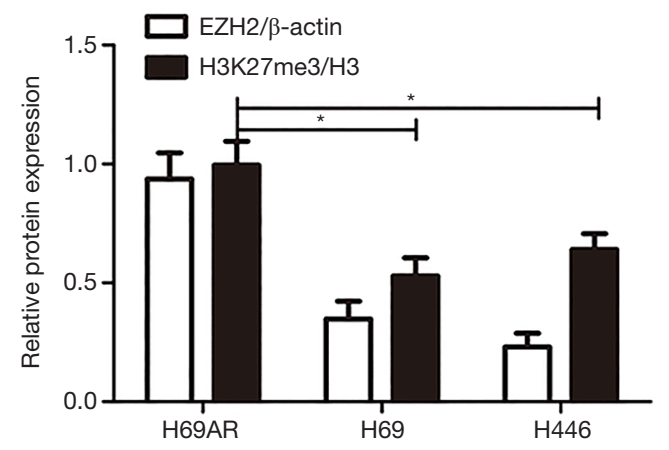

C

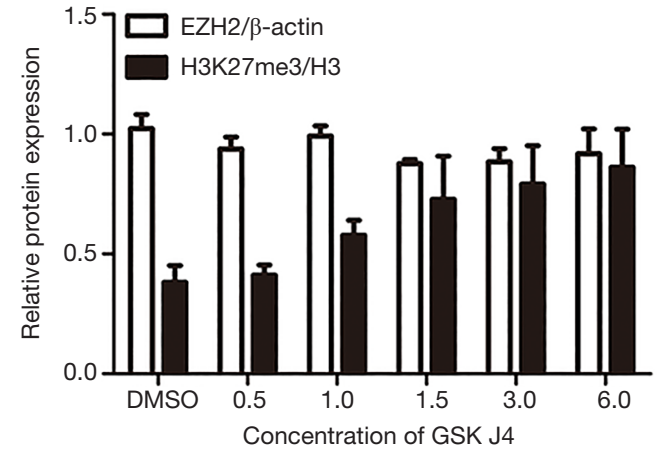

B

H69AR

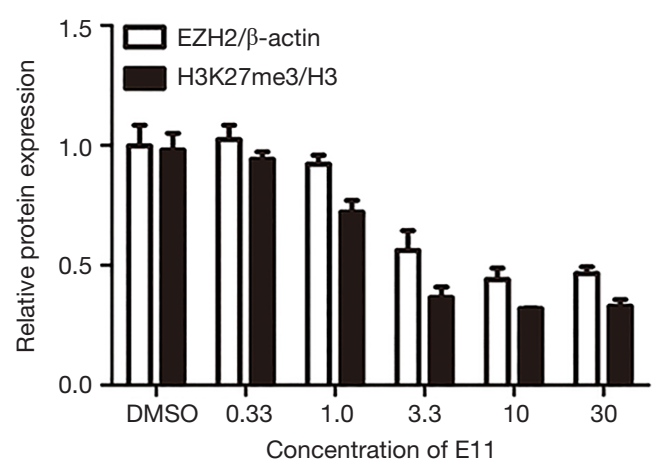

$\mathrm{H} 446$

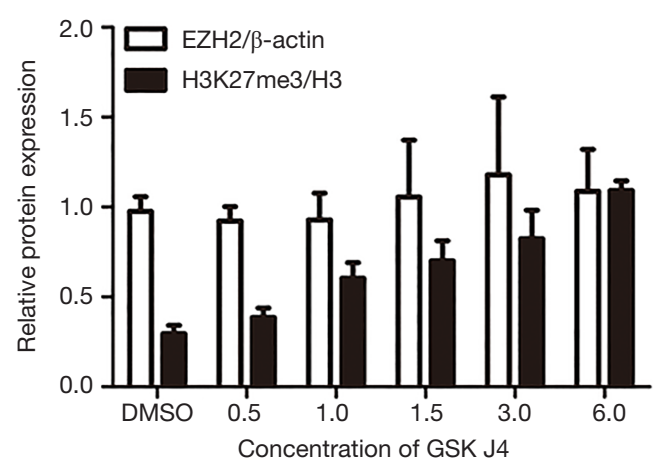

Figure S2 Protein levels of EZH2 and increased H3K27me3 were revealed by Western blotting in SCLC cells and after treatment with E1I and GSK J4. Band intensities were measured by densitometry; EZH2 levels were normalized to $\beta$-actin expression, and H3K27me3 levels were normalized to $\mathrm{H} 3$ expression.
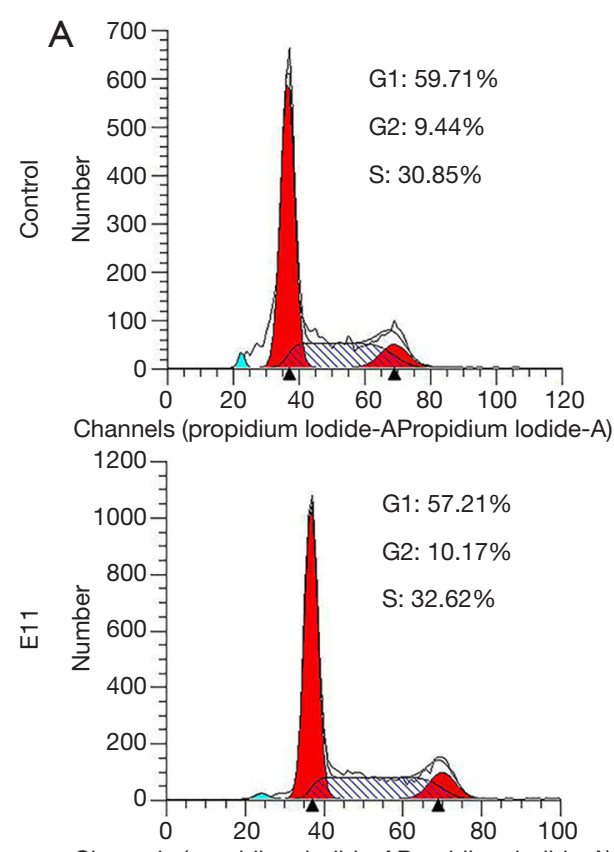

Channels (propidium lodide-APropidium lodide-A)
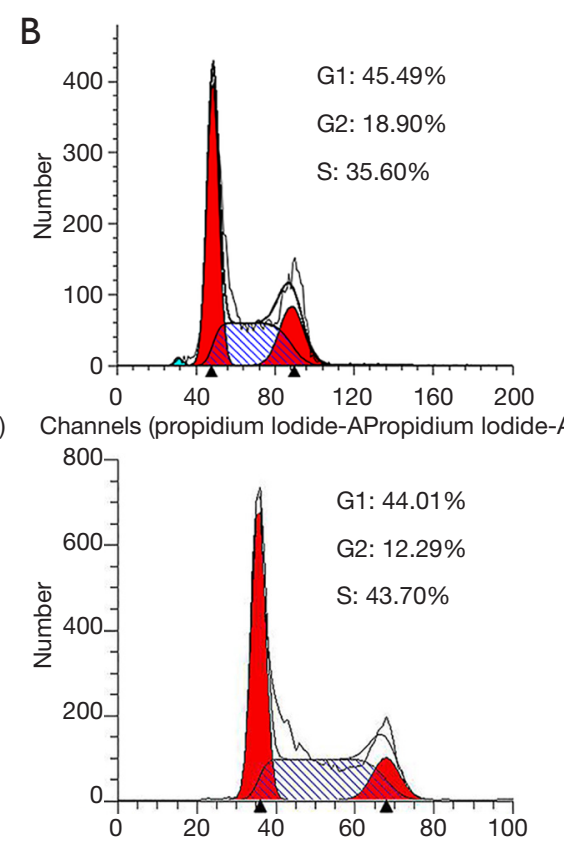

Channels (propidium lodide-APropidium lodide-A)
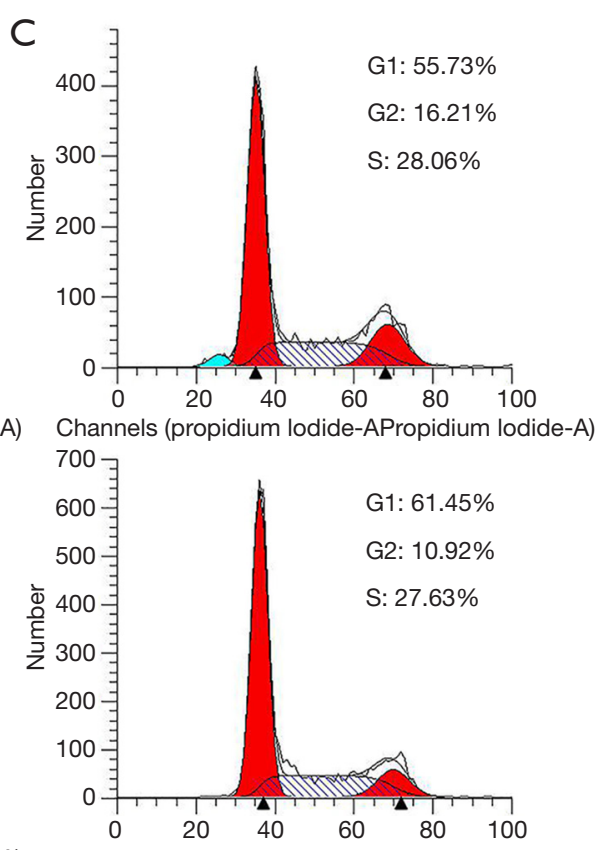

Channels (propidium lodide-APropidium lodide-A)

Figure S3 Cell cycle progression analyses did not indicate significant differences. Cell cycle progression was determined by flow cytometry after H69AR cells were exposed to CDDP (A), VP-16 (B) and ADM (C). 